\title{
A Representation of all Solutions of the Control Algebraic Riccati Equation for Infinite-Dimensional Systems
}

\author{
Orest V. Iftime*, Hans Zwart† Ruth F. Curtain*
}

\begin{abstract}
We obtain a representation of all self-adjoint solutions of the control algebraic Riccati equation associated to the infinite-dimensional state linear system $\Sigma(A, B, C)$ under the following assumptions: $A$ generates a $C_{0}$-group, the system is output stabilizable, strongly detectable and the dual Riccati equation has an invertible selfadjoint nonnegative solution.
\end{abstract}

*University of Groningen, Department of Mathematics and Computing Science, PO Box 800, 9700 AV Groningen, The Netherlands. Tel.: +31 (0)50 363 6496. Fax: +31 (0)50 3633800. Email: R.F.Curtain@math.rug.nl, O.V.Iftime@math.rug.nl

†University of Twente, Department of Applied Mathematics, Faculty of EEMCS, P.O. Box 217 7500 AE Enschede, The Netherlands. Tel.: +31 (0)53 489 3464. Fax: +31 (0)53 489 3800. Email: h.j.zwart@math.utwente.nl 


\section{Introduction}

In finite-dimensional systems theory the control algebraic Riccati equation (CARE)

$$
A^{*} X+X A-X B B^{*} X+C^{*} C=0
$$

is a successful tool for solving various problems, for example, optimal control, optimal filtering, stability analysis et al. (see e.g. [4, 7, 20, 21], and the references therein). An overview of important results on Riccati theory for finite-dimensional systems has been gathered by Bittanti, Laub and Willems [2].

Since the CARE is such an effective tool in finite-dimensional control theory, more and more effort has been made in extending these results to infinite-dimensional systems, see e.g. $[3,5,6,12,17]$, and the references therein. Recently, a very general theory of optimal control problems and the CARE was developed by Mikkola in [13].

In [21], Willems gave a classification of all solutions of the CARE for finite-dimensional systems. These results increased the range of applications of the Riccati theory significantly. In this paper we show that the classification of all solutions of the CARE may be extended to a class of strongly stabilizable and detectable infinite-dimensional systems. Earlier papers in this direction considered more restrictive or different assumptions. Under the spectrum decomposition assumption (for exponentially stabilizable, infinitedimensional systems) Callier et al. [3] gave a classification of all nonnegative self-adjoint solutions of the CARE. A classification of all nonnegative self-adjoint solutions of the CARE for discrete-time systems was found by Malinen in [12]. However, we are interested in all solutions and not just the nonnegative ones.

Another approach to characterize the solutions of the CARE is to use the Hamiltonian operator, see e.g. Adamjam et al. [1], Kuiper and Zwart [10] and Langer et al. [11]. In [1] the special case that the Hamiltonian operator is Riesz spectral was considered. In other papers a standard assumption is that the Hamiltonian operator is dichotomous, i.e. there is a strip around the imaginary axis which is contained in the resolvent set of the Hamiltonian operator. Under our set of assumptions this need not hold.

In this paper we obtain a representation of all self-adjoint solutions to the CARE corresponding to the state linear system $\Sigma(A, B, C)$ under the following assumptions

- $A$ generates a $C_{0}$-group $T(t)$ on a separable Hilbert space $Z$;

- $\Sigma(A, B, C)$ is output stabilizable;

- $\Sigma(A, B, C)$ is strongly detectable; 
- The minimal self-adjoint nonnegative solution of the filter algebraic Riccati equation (FARE) is invertible.

Under the first three assumptions, there exists a unique self-adjoint nonnegative solution $X^{+}:=X_{o p t}$ of the CARE and a minimal nonnegative self-adjoint solution $Y_{\text {opt }}$ of the FARE. Adding the assumption that $A$ generates a $C_{0}$-group, we obtain that $Y^{+}$, the maximal selfadjoint solution of the FARE, is equal to $Y_{\text {opt }}$. Moreover, $X^{-}:=-\left(Y^{+}\right)^{-1}$ is the minimal self-adjoint solution of the CARE. We prove that every self-adjoint solution $X \in \mathcal{L}(Z)$ of the CARE satisfies $X^{-} \leq X \leq X^{+}$. Furthermore, we obtain the following generalization to this class of infinite-dimensional systems of an important result from the well-known paper of Willems [21].

Theorem 1.1. Under the above assumptions, every self-adjoint $X \in \mathcal{L}(Z)$ is a solution of the CARE if and only if $X$ can be decomposed as

$$
X=X^{+} P+X^{-}(I-P)
$$

for a certain projection $P \in \mathcal{L}(Z)$.

The precise formulation of the above result is stated in Theorem 4.15. There we indicate precisely which projections provide representations for all self-adjoint solutions of the CARE.

Finally, we comment on the limitation that $A$ generates a $C_{0}$-group. Sufficient conditions for the $C_{0}$-semigroup $T(t)$ to be embedded in a $C_{0}$-group can be found, for example, in [16] (see Theorem 6.5 and Theorem 6.6 on page 24) and [22]. While this is clearly a restriction, there does exist an important class of systems that satisfies our assumptions. This is the class of dissipative systems with collocated actuators and sensors, denoted by $\Sigma\left(A, B, B^{*}\right)$, where $A$ is skew-adjoint $\left(D(A)=D\left(A^{*}\right)\right.$ and $\left.A+A^{*}=0\right)$. Although mathematically very special, they have a prominent place in the engineering literature, mainly as models for flexible structures. For an extensive overview we refer to Chapter 9 in [14].

\section{Preliminaries}

We consider a state linear system $\Sigma(A, B, C)$ of the form

$$
\begin{aligned}
\dot{z}(t) & =A z(t)+B u(t), \quad z(0)=z_{0} \in Z \\
y(t) & =C z(t)
\end{aligned}
$$


with $z \in Z, y \in Y$ and $u \in U$, where $Z, Y$ and $U$ are separable Hilbert spaces, $B \in \mathcal{L}(U, Z)$ and $C \in \mathcal{L}(Z, Y)$, i.e. $B$ and $C$ are bounded operators. The operator $A: \mathcal{D}(A) \subset Z \rightarrow Z$ is the infinitesimal generator of the $C_{0}$-semigroup $T(t)$ of bounded operators on $Z$. The state and the output trajectories of the system $\Sigma(A, B, C)$ are given by

$$
\begin{aligned}
& z(t)=T(t) z_{0}+\int_{0}^{t} T(t-s) B u(s) d s \\
& y(t)=C z(t),
\end{aligned}
$$

where $z(0)=z_{0} \in Z$. As the cost functional for this system, we take

$$
J_{[0, t]}\left(z_{0}, u\right)=\int_{0}^{t}\left(\|y(s)\|^{2}+\|u(s)\|^{2}\right) d s
$$

where $u$ is the input trajectory and $t$ is a positive real number or infinity.

We recall the concepts of stability and strong stability of the linear system $\Sigma(A, B, C)$. The definition of a strongly stable linear system was introduced by Staffans $[18,19]$.

Definition 2.1. The state linear system $\Sigma(A, B, C)$ is a stable system if

- it is input stable, i.e., there exists a constant $\beta>0$ such that for all $u \in L_{2}(0, \infty ; U)$

$$
\left\|\int_{0}^{\infty} T(t) B u(t) d t\right\|^{2} \leq \beta \int_{0}^{\infty}\|u(t)\|^{2} d t
$$

- it is output stable, i.e., there exists a constant $\gamma>0$ such that for all $z \in Z$

$$
\int_{0}^{\infty}\|C T(t) z\|^{2} d t \leq \gamma\|z\|^{2}, \text { and }
$$

- it is input-output stable, i.e., the transfer function $C(s I-A)^{-1} B$ is an element of $H_{\infty}(\mathcal{L}(U, Y))$, the space of bounded holomorphic functions in the open right half plane.

The state linear system $\Sigma(A, B, C)$ is a strongly stable system if it is stable and it satisfies

- A generates a strongly stable semigroup, i.e., for all $z_{0} \in Z, T(t) z_{0} \rightarrow 0$ as $t \rightarrow \infty$.

The properties input and output stability of a linear system are related to the existence of solutions to Lyapunov equations, see [8] and [9]. 
Lemma 2.2. The state linear system $\Sigma(A, B, C)$ is output stable if and only if the following observability Lyapunov equation has a self-adjoint nonnegative solution $L \in \mathcal{L}(Z)$ :

$$
A^{*} L+L A=-C^{*} C \text { on } D(A) \text {. }
$$

Lemma 2.3. The state linear system $\Sigma(A, B, C)$ is input stable if and only if the following controllability Lyapunov equation has a self-adjoint nonnegative solution $L \in \mathcal{L}(Z)$ :

$$
A L+L A^{*}=-B B^{*} \text { on } D\left(A^{*}\right) .
$$

In addition to the definition of a strongly stable system we also need the related notions of stabilizability and detectability. For convenience, we use $[F ; C]$ to denote the column matrix constructed from $F$ and $C$.

Definition 2.4. The system $\Sigma(A, B, C)$ is strongly stabilizable if

- it is output stabilizable, i.e., there exists an $F \in \mathcal{L}(Z, U)$ such that $\Sigma(A+B F, B,[F ; C])$ is output stable, and

- A+BF generates a strongly stable semigroup.

Definition 2.5. The system $\Sigma(A, B, C)$ is strongly detectable if

- it is input stabilizable, i.e., there exists an $L \in \mathcal{L}(Y, Z)$ such that $\Sigma(A+L C,[L, B], C)$ is input stable, and

- $A+L C$ generates a strongly stable semigroup.

We recall that the control algebraic Riccati equation (CARE) is

$$
A^{*} X+X A-X B B^{*} X+C^{*} C=0 \text { on } D(A)
$$

and the filter algebraic Riccati equation (FARE) is

$$
A Y+Y A^{*}-Y C^{*} C Y+B B^{*}=0 \text { on } D\left(A^{*}\right)
$$

If $X$ and $Y$ are solutions for the CARE and FARE we introduce the following notations

$$
A_{X}:=A-B B^{*} X, \text { and } A_{Y}:=A-Y C^{*} C
$$

By $T_{X}(t)$ and $T_{Y}(t)$ we denote the $C_{0}$-semigroups generated by $A_{X}$ and $A_{Y}$, respectively. The following result establishes a link between a self-adjoint solution of the CARE and 
the finite horizon control problem (this may be proved as in [6], see Lemma 6.2.3, or see Lemma B.2).

Lemma 2.6. Suppose that $X \in \mathcal{L}(Z)$ is a solution of the $C A R E$. Then for all $t \geq 0$, for all $u \in L_{2}(0, t ; U)$, and for all $z_{0} \in Z$ the following holds:

$$
J_{[0, t]}\left(z_{0}, u\right)=\int_{0}^{t}\left\|u(s)+B^{*} X z(s)\right\|^{2} d s+\left\langle z_{0}, X z_{0}\right\rangle-\langle z(t), X z(t)\rangle
$$

where $z(\cdot)$ is a trajectory of the linear system and the cost function $J_{[0, t]}\left(z_{0}, u\right)$ is defined by (4).

We recall the notion of an invariant subspace of a Hilbert space. If $T$ is an operator on a Hilbert space $Z$, and if $V$ is a linear subspace which satisfies $T V \subseteq V$, then $V$ is called an invariant subspace of $Z$ with respect to $T$. We consider two types of invariance, namely semigroup-invariance and invariance with respect to the infinitesimal generator.

Definition 2.7. Let $V$ be a subspace of the Hilbert space $Z$ and let $T(t)$ be a $C_{0}$-semigroup on $Z$. We say that $V$ is $T(t)$-invariant if for all $t \geq 0$

$$
T(t) V \subseteq V
$$

Definition 2.8. Let $V$ be a subspace of the Hilbert space $Z$ and let $A$ be an infinitesimal generator of a $C_{0}$-semigroup on $Z$. We say that $V$ is $A$-invariant if

$$
A(V \cap \mathcal{D}(A)) \subseteq V
$$

\section{Existence of the solutions of the AREs and stability properties of the closed-loop system}

In this section we provide sufficient conditions for the existence of solutions for CARE and FARE. Moreover, stability properties of the closed-loop system are analyzed.

First, we prove that output stabilizability implies the existence of a minimal self-adjoint nonnegative solution of the CARE. Moreover, the corresponding closed-loop system is output stable and input-output stable. These results are extensions of the results presented in $[14$, Theorem 3.3.2(1)]. 
Theorem 3.1. If the linear system $\Sigma(A, B, C)$ is output stabilizable, then there exists $X_{\text {opt }}$, the minimal self-adjoint nonnegative solution of the CARE. Moreover, the system $\Sigma\left(A_{X_{\text {opt }}}, B,\left[-B^{*} X_{\text {opt }} ; C\right]\right)$ is output stable and input-output stable.

Proof. We prove first that output stabilizability implies optimizability, see [6, Definition 6.2.1]. Since the linear system $\Sigma(A, B, C)$ is output stabilizable, there exists an $F \in$ $\mathcal{L}(Z, U)$ such that $\Sigma(A+B F, B,[F ; C])$ is output stable. Consequently, by taking the input $u(t)=F z(t)$, we have that there exists a constant $c>0$ such that for every $z_{0} \in Z$

$$
J_{[0, \infty)}\left(z_{0}, F z\right)=\int_{0}^{\infty}\left(\left\|C T_{F}(t) z_{0}\right\|^{2}+\left\|F T_{F}(t) z_{0}\right\|^{2}\right) d t \leq c\left\|z_{0}\right\|^{2} .
$$

So, the system $\Sigma(A, B, C)$ is optimizable. The conditions of Theorem 6.2.4 in [6] are satisfied, and there exists a minimal self-adjoint nonnegative solution $X_{\text {opt }}$ of the CARE.

From the definition of the cost functional and [6, Theorem 6.2.4], with the optimal control given by $u(t)=-B^{*} X_{o p t} T_{X_{o p t}}(t) z_{0}$, we have that

$$
\left\langle z_{0}, X_{\text {opt }} z_{0}\right\rangle=\int_{0}^{\infty}\left(\left\|C T_{X_{o p t}}(t) z_{0}\right\|^{2}+\left\|B^{*} X_{o p t} T_{X_{o p t}}(t) z_{0}\right\|^{2}\right) d t
$$

In particular, for every $z_{0} \in Z$ the following inequalities hold

$$
\int_{0}^{\infty}\left\|C T_{X_{o p t}}(t) z_{0}\right\|^{2} d t<\infty
$$

and

$$
\int_{0}^{\infty}\left\|B^{*} X_{o p t} T_{X_{o p t}}(t) z_{0}\right\|^{2} d t<\infty
$$

This implies that $\Sigma\left(A_{X_{o p t}}, B,\left[-B^{*} X_{o p t} ; C\right]\right)$ is output stable.

Further, we prove that the linear system $\Sigma\left(A_{X_{o p t}}, B,\left[-B^{*} X_{o p t} ; C\right]\right)$ is input-output stable. We start by rewriting the CARE, for $z_{1} \in D(A)$, as

$$
A_{X_{o p t}}^{*} X_{o p t} z_{1}+X_{o p t} A_{X_{o p t}} z_{1}+X_{o p t} B B^{*} X_{o p t} z_{1}+C^{*} C z_{1}=0 .
$$

Taking the inner product with $z_{2} \in D(A)$ we obtain

$$
\left\langle X_{o p t} z_{1}, A_{X_{o p t}} z_{2}\right\rangle+\left\langle A_{X_{o p t}} z_{1}, X_{o p t} z_{2}\right\rangle+\left\langle\widetilde{C} z_{1}, \widetilde{C} z_{2}\right\rangle=0
$$


where $\tilde{C}:=\left[-B^{*} X_{\text {opt }} ; C\right]$. For $u_{1}, u_{2} \in C^{1}(0, t ; U)$, define

$$
z_{i}(t):=\mathcal{B}^{t} u_{i}=\int_{0}^{t} T_{X_{o p t}}(t-s) B u_{i}(s) d s, i=1,2 .
$$

By Theorem 3.1.3 in [6], $z_{i}(s) \in D(A)$ for all $s \geq 0$ and $i=1,2$, and it is the classical solution, i.e.,

$$
\dot{z}_{i}(s)=A_{X_{o p t}} z_{i}(s)+B u_{i}(s), \quad z_{i}(0)=0, i=1,2 .
$$

Note that $\mathcal{B}^{\tau}$ is the controllability map of the system $\Sigma\left(A_{X_{o p t}}, B,\left[-B^{*} X_{o p t} ; C\right],[I ; 0]\right)$. Substituting $z_{i}(s)$ in the Riccati equation gives

$$
\frac{d}{d t}\left\langle X_{o p t} z_{1}(s), z_{2}(s)\right\rangle=\left\langle u_{1}(s), B^{*} X_{o p t} z_{2}(s)\right\rangle+\left\langle B^{*} X_{o p t} z_{1}(s), u_{2}(s)\right\rangle-\left\langle\widetilde{C} z_{1}(s), \widetilde{C} z_{2}(s)\right\rangle .
$$

Integrating from 0 to $t$ gives

$$
\begin{aligned}
\left\langle X_{o p t} z_{1}(t), z_{2}(t)\right\rangle= & \int_{0}^{t}\left\langle u_{1}(s), B^{*} X_{o p t} z_{2}(s)\right\rangle d s+\int_{0}^{t}\left\langle B^{*} X_{o p t} z_{1}(s), u_{2}(s)\right\rangle d s \\
& -\int_{0}^{t}\left\langle\widetilde{C} z_{1}(s), \widetilde{C} z_{2}(s)\right\rangle d s
\end{aligned}
$$

The input-output map $\mathcal{D}^{t}$ of the state linear system $\Sigma\left(A_{X_{\text {opt }}}, B, \widetilde{C},[I ; 0]\right)$ is given by

$$
\left(\mathcal{D}^{t} u\right)(\alpha):=[u(\alpha) ; 0]+\int_{0}^{\alpha} \tilde{C} T_{X_{o p t}}(\alpha-s) B u(s) d s
$$

where $\alpha \in(0, t)$ and $u \in L_{2}(0, \tau ; U)$. Now

$$
\begin{aligned}
\left\langle\mathcal{D}^{t} u_{1}, \mathcal{D}^{t} u_{2}\right\rangle= & \left\langle\left[u_{1}(t) ; 0\right]+\widetilde{C} z_{1}(t),\left[u_{2}(t) ; 0\right]+\widetilde{C} z_{2}(t)\right\rangle \\
= & \left\langle u_{1}(t), u_{2}(t)\right\rangle-\left\langle u_{1}(t), B^{*} X_{o p t} z_{2}(t)\right\rangle \\
& -\left\langle B^{*} X_{o p t} z_{1}(t), u_{2}(t)\right\rangle+\left\langle\widetilde{C} z_{1}(t), \widetilde{C} z_{2}(t)\right\rangle .
\end{aligned}
$$

Using equation (9) we see that

$$
\left\langle X_{o p t} z_{1}(t), z_{2}(t)\right\rangle+\int_{0}^{t}\left\langle\left(\mathcal{D}^{t} u_{1}\right)(s),\left(\mathcal{D}^{t} u_{2}\right)(s)\right\rangle d s=\int_{0}^{t}\left\langle u_{1}(s), u_{2}(s)\right\rangle d s,
$$

for all $u_{1}, u_{2} \in C^{1}(0, t ; U)$. In order to show that this can be extended to all locally $L_{2}(0, t ; U)$ inputs one can approximate functions $u_{1}, u_{2} \in L_{2}(0, t ; U)$ with $u_{1}^{n}, u_{2}^{n} \in C^{1}(0, t ; U)$. Then (using standard techniques) it can be proved that it is possible to take the limit as 
$n \rightarrow \infty$ in equality (10).

Choosing in equation (10) $u_{1}=u_{2}$ and letting $t \rightarrow \infty$ we see that $\mathcal{D}^{\infty}$ is a bounded linear operator and the linear system $\Sigma\left(A_{X_{\text {opt }}}, B,\left[-B^{*} X_{\text {opt }} ; C\right],[I ; 0]\right)$ is input-output stable. Consequently, the linear system $\Sigma\left(A_{X_{o p t}}, B,\left[-B^{*} X_{o p t} ; C\right]\right)$ is input-output stable.

Because the dual result of Theorem 3.1 is completely analogous (by duality), we state it without a proof.

Theorem 3.2. If the state linear system $\Sigma(A, B, C)$ is input stabilizable, then there exists $Y_{\text {opt }}$, a minimal self-adjoint nonnegative solution of the FARE. Moreover, the state linear system $\Sigma\left(A_{Y_{\text {opt }}},\left[B,-Y_{\text {opt }} C^{*}\right], C\right)$ is input stable and input-output stable.

If one assumes both input and output stabilizability of the linear system $\Sigma(A, B, C)$, then the stability of the corresponding closed-loop system is obtained.

Theorem 3.3. If the linear system $\Sigma(A, B, C)$ is input and output stabilizable, then $\Sigma\left(A_{X_{\text {opt }}}, B,\left[-B^{*} X_{\text {opt }} ; C\right]\right)$ is a stable system, where $X_{\text {opt }}$ is the minimal self-adjoint nonnegative solution of the CARE.

Proof. From Theorem 3.1 we have that the linear system $\Sigma\left(A_{X_{\text {opt }}}, B,\left[-B^{*} X_{\text {opt }} ; C\right]\right)$ is output stable and input-output stable. In order to prove that it is also input stable we will use Lemma 2.3, i.e., we have to prove that the controllability Lyapunov equation

$$
A_{X_{o p t}} L+L A_{X_{o p t}}^{*}=-B B^{*} \text {, on } D\left(A^{*}\right)
$$

has a self-adjoint nonnegative solution.

The input and output stabilizability guarantee the existence of a nonnegative selfadjoint solution $Y_{o p t}$ to the FARE (see Theorem 3.2) and a nonnegative self-adjoint solution $X_{o p t}$ to the CARE. Then $\left(I+Y_{o p t} X_{o p t}\right)$ is boundedly invertible and $\left(I+Y_{o p t} X_{o p t}\right)^{-1} Y_{\text {opt }}$ is non-negative. It remains to show that it is a solution of (11).

From equation (51) we have that on $D(A)$

$$
\left(I+Y_{\text {opt }} X_{\text {opt }}\right)^{-1}\left(A-Y_{\text {opt }} C^{*} C\right)=\left(A-B B^{*} X_{\text {opt }}\right)\left(I+Y_{\text {opt }} X_{\text {opt }}\right)^{-1}
$$

Since $Y_{\text {opt }} D\left(A^{*}\right) \subset D(A)$, this implies that

$$
\left(I+Y_{o p t} X_{o p t}\right)^{-1}\left(A-Y_{o p t} C^{*} C\right) Y_{o p t}=\left(A-B B^{*} X_{o p t}\right)\left(I+Y_{o p t} X_{o p t}\right)^{-1} Y_{o p t} \quad \text { on } D\left(A^{*}\right)
$$


Using the FARE, we obtain

$$
\left(I+Y_{\text {opt }} X_{\text {opt }}\right)^{-1}\left(-Y_{\text {opt }} A^{*}-B B^{*}\right)=\left(A-B B^{*} X_{\text {opt }}\right)\left(I+Y_{\text {opt }} X_{\text {opt }}\right)^{-1} Y_{\text {opt }} \quad \text { on } D\left(A^{*}\right) .
$$

So on $D\left(A^{*}\right)$ we find

$$
\begin{aligned}
(A-B & \left.B{ }^{*} X_{o p t}\right)\left(I+Y_{o p t} X_{o p t}\right)^{-1} Y_{o p t} \\
& =\left(I+Y_{o p t} X_{o p t}\right)^{-1}\left(-Y_{o p t} A^{*}-B B^{*}\right) \\
& =\left(I+Y_{o p t} X_{o p t}\right)^{-1}\left(-Y_{o p t} A^{*}+Y_{o p t} X_{o p t} B B^{*}-Y_{o p t} X_{o p t} B B^{*}-B B^{*}\right) \\
& =-B B^{*}+\left(I+Y_{o p t} X_{o p t}\right)^{-1} Y_{o p t}\left(-A^{*}+X_{o p t} B B^{*}\right) .
\end{aligned}
$$

This shows that $\left(I+Y_{\text {opt }} X_{\text {opt }}\right)^{-1} Y_{\text {opt }}$ is the solution of (11).

A dual version of Theorem 3.3 can be proved analogously.

Theorem 3.4. If the linear system $\Sigma(A, B, C)$ is input and output stabilizable, then $\Sigma\left(A_{Y_{\text {opt }}},\left[B-Y_{\text {opt }} C^{*}\right], C\right)$ is a stable system, where $Y_{\text {opt }}$ is the minimal self-adjoint nonnegative solution of the FARE.

Statements about the uniqueness of the solutions of the Riccati equations can be obtained by adding conditions for strong stability.

Theorem 3.5. If the linear system $\Sigma(A, B, C)$ is output stabilizable and strongly detectable, then $X_{\text {opt }}$ is the unique self-adjoint nonnegative solution of the CARE and the state linear system $\Sigma\left(A_{X_{\text {opt }}}, B,\left[-B^{*} X_{\text {opt }} ; C\right]\right)$ is strongly stable.

Proof. Strong detectability implies input stabilizability. We can apply Theorem 3.3 and the second part of Theorem 3.3.2 from [14] to complete the proof.

Theorem 3.6. If the state linear system $\Sigma(A, B, C)$ is input stabilizable and output stabilizable, i.e., there exists an $F$ such that $(A+B F, B,[F ; C])$ is output stable. Then the state linear system $\Sigma\left(A_{Y_{o p t}},\left[B-Y_{\text {opt }} C^{*}\right], C\right)$ is stable.

Furthermore, if $(A+B F)^{*}$ generates a strongly stable semigroup, then $\left(A_{Y_{\text {opt }}}\right)^{*}$ generates a strongly stable semigroup and $Y_{\text {opt }}$ is the unique self-adjoint nonnegative solution of the FARE

Proof. The result is a consequence of Theorem 3.4 and the proof of the second part of Theorem 3.3.4 in [14]. 


\section{A classification of all self-adjoint solutions of the CARE}

\subsection{Assumptions and their consequences}

We begin by making some additional assumptions on the state linear system $\Sigma(A, B, C)$.

Assumption 4.1. The state linear system $\Sigma(A, B, C)$ is output stabilizable.

Assumption 4.2. The state linear system $\Sigma(A, B, C)$ is strongly detectable.

Remark 4.1. Under the Assumptions 4.1 and 4.2, Theorem 3.5 guarantees that $X_{\text {opt }}$ is the unique self-adjoint nonnegative solution of the CARE. Consequently it is also the maximal one, and, for the sake of simplicity, we make the notation $X^{+}:=X_{\text {opt }}$. Moreover, $X^{+}$is a strongly stabilizable solution of the $C A R E$, i.e., the linear system $\Sigma\left(A_{X^{+}}, B,\left[-B^{*} X^{+} ; C\right]\right)$ is a strongly stable system.

Remark 4.2. Strong detectability implies input stabilizability (see Definition 2.5) of the state linear system $\Sigma(A, B, C)$. Consequently, Theorem 3.2 provides the existence of a minimal nonnegative self-adjoint solution $Y_{\text {opt }}$ of the FARE.

We now make two additional assumptions on $Y_{\text {opt }}$ and $A$, respectively.

Assumption 4.3. The minimal nonnegative self-adjoint solution of the FARE associated to the linear system $\Sigma(A, B, C)$, $Y_{\text {opt }}$, is invertible.

Assumption 4.4. A generates a $C_{0}$-group.

Assumptions 4.2-4.3 have the following consequences.

Lemma 4.3. If Assumptions 4.2 and 4.3 hold, then $Y_{\text {opt }}$ is the unique nonnegative selfadjoint solution of the FARE, and $A_{Y_{\text {opt }}}^{*}$ generates a strongly stable $C_{0}$-semigroup, $T_{Y_{\text {opt }}}^{*}(t)$.

Proof. Using a dual version of Lemma 2.6 and the fact that $Y_{\text {opt }}$ is the minimal nonnegative self-adjoint solution of the FARE, we have that $\left\langle z(t), Y_{\text {opt }} z(t)\right\rangle \rightarrow 0$, for $t \rightarrow \infty$, where $z(t)=T_{Y_{\text {opt }}}^{*}(t) z_{0}$. Since $Y_{\text {opt }}$ is invertible, it follows that $z(t) \rightarrow 0$ for $t \rightarrow \infty$, or equivalently $T_{Y_{\text {opt }}}^{*}(t)$ is a strongly stable $C_{0}$-semigroup. Hence $Y_{\text {opt }}$ is a strongly stabilizing solution of the FARE, and thus it is the maximal nonnegative self-adjoint solution (this follows as in Lemma 6.2.6 in [6]). By assumption it is also the minimal one, and thus it is unique. 
Remark 4.4. We used the invertibility of $Y_{\text {opt }}$ to prove that $A_{Y_{o p t}}^{*}$ generates a strongly stable $C_{0}$-semigroup, $T_{Y_{\text {opt }}}^{*}(t)$. The uniqueness of $Y_{\text {opt }}$ follows in exactly the same way as in the case of an exponentially stable semigroup, the dual versions of the proofs of Lemma 6.2.6 and Theorem 6.2.7 in [6].

Remark 4.5. Since $Y_{\text {opt }}$ is the unique nonnegative self-adjoint solution of the FARE, it is also the maximal one. In the sequel we use the notation $Y^{+}:=Y_{\text {opt }}$ and $A_{Y^{+}}:=A-Y^{+} C^{*} C$.

Using Assumption 4.4, we prove that $-\left(Y^{+}\right)^{-1}$ is a nonpositive solution of the CARE. In fact, we obtain the following equivalent statements.

Lemma 4.6. Suppose that the state linear system $\Sigma(A, B, C)$ satisfies the Assumptions 4.1-4.3. Then the following statements are equivalent:

1. A generates a $C_{0}$-group, i.e., Assumption 4.4 is satisfied;

2. $Y^{+} D\left(A^{*}\right)=D(A)$;

3. $X^{-}:=-\left(Y^{+}\right)^{-1}$ is a solution of the CARE.

Proof. $\mathbf{1} \Rightarrow \mathbf{2}$ : By Remark 4.5 we have that $Y^{+}$is a self-adjoint invertible solution of the FARE. Furthermore, by defining $Q:=-Y^{+} C^{*} C Y^{+}+B B^{*}$, we can write the FARE as

$$
A Y^{+}+Y^{+} A^{*}=-Q
$$

By assumption we have that $A$ generates a $C_{0}$-group. Using Theorem 3.2.1 in [6], we have that $-A_{Y^{+}}-Q\left(Y^{+}\right)^{-1}$ generates a $C_{0^{-}}$group as well. Exercise 2.19.d from [6] implies that $-A_{Y^{+}}-Q\left(Y^{+}\right)^{-1}$ has its spectrum in a strip along the imaginary axis, and so $\rho\left(-A_{Y^{+}}-\right.$ $\left.Q\left(Y^{+}\right)^{-1}\right) \cap \rho\left(A_{Y^{+}}^{*}\right) \neq \emptyset$. Using Corollary A.2, we conclude that $Y^{+} D\left(A^{*}\right)=D(A)$.

2 $\Rightarrow$ 3: Suppose that $Y^{+} D\left(A^{*}\right)=D(A)$. Consider arbitrary $x, y \in D(A)$. Then, there exist $x_{0}, y_{0} \in D\left(A^{*}\right)$ such that $x=Y^{+} x_{0}$ and $y=Y^{+} y_{0}$. We have the following sequence of equalities

$$
\begin{aligned}
\left\langle A^{*} X^{-}\right. & x, y\rangle+\left\langle X^{-} A x, y\right\rangle-\left\langle X^{-} B B^{*} X^{-} x, y\right\rangle+\left\langle C^{*} C x, y\right\rangle \\
& =\left\langle-A^{*}\left(Y^{+}\right)^{-1} x, y\right\rangle+\left\langle-\left(Y^{+}\right)^{-1} A x, y\right\rangle-\left\langle\left(Y^{+}\right)^{-1} B B^{*}\left(Y^{+}\right)^{-1} x, y\right\rangle+\left\langle C^{*} C x, y\right\rangle \\
& =-\left\langle\left(Y^{+}\right)^{-1} x, A y\right\rangle-\left\langle A x,\left(Y^{+}\right)^{-1} y\right\rangle-\left\langle B^{*}\left(Y^{+}\right)^{-1} x, B^{*}\left(Y^{+}\right)^{-1} y\right\rangle+\langle C x, C y\rangle \\
& =-\left\langle x_{0}, A Y^{+} y_{0}\right\rangle-\left\langle A Y^{+} x_{0}, y_{0}\right\rangle-\left\langle B^{*} x_{0}, B^{*} y_{0}\right\rangle+\left\langle C Y^{+} x_{0}, C Y^{+} y_{0}\right\rangle \\
& =-\left(\left\langle Y^{+} A^{*} x_{0}, y_{0}\right\rangle+\left\langle A Y^{+} x_{0}, y_{0}\right\rangle-\left\langle Y^{+} C^{*} C Y^{+} x_{0}, y_{0}\right\rangle+\left\langle B B^{*} x_{0}, y_{0}\right\rangle\right)=0 .
\end{aligned}
$$


Since $x, y \in D(A)$ are arbitrary, we conclude that $X^{-}$is a solution of the CARE.

$\mathbf{3} \Rightarrow \mathbf{2}$ : Since $Y^{+}$and $-\left(Y^{+}\right)^{-1}$ are solutions of the FARE and CARE, respectively, we have that $Y^{+} D\left(A^{*}\right) \subset D(A)$ and $\left(Y^{+}\right)^{-1} D(A) \subset D\left(A^{*}\right)$. This shows that $Y^{+} D\left(A^{*}\right)=D(A)$.

$\mathbf{2} \Rightarrow \mathbf{1}$ : We know that $A$ generates a $C_{0}$-semigroup. From Theorem 3.2.1 and Theorem 2.2.6 in [6] we have that $A_{X^{+}}^{*}$ generates a $C_{0^{-}}$-semigroup. Since Assumption 4.1-4.3 are satisfied and $Y^{+} D\left(A^{*}\right)=D(A)$, we obtain from Lemma A.8 that

$$
-A_{X^{-}}=-A+B B^{*}\left(-Y^{+}\right)^{-1}=\Delta^{-1}\left(A_{X^{+}}\right)^{*} \Delta
$$

holds on $D(A)$, where $\Delta:=X^{+}+\left(Y^{+}\right)^{-1}$. Furthermore, using Exercise 2.5 and Theorem 3.2.1 from [6] we conclude that $-A$ generates a $C_{0}$-semigroup. Finally, from Exercise 2.19.c in [6] we obtain that $A$ generates a $C_{0}$-group.

Corollary 4.7. If Assumptions 4.1-4.4 are satisfied, then $X^{-}:=-\left(Y^{+}\right)^{-1}$ is the maximal nonpositive, self-adjoint solution of the CARE of the original system.

Using the following lemma, we are able to prove the uniqueness of the self-adjoint, nonpositive solution of the CARE.

Lemma 4.8. Suppose that the state linear system $\Sigma(A, B, C)$ satisfies Assumptions 4.14.4. Then $T_{X^{-}}(-t)$ (the semigroup generated by $\left.-A_{X^{-}}\right)$and $T_{X^{+}}^{*}(t)$ are strongly stable semigroups.

Proof. Using Lemma 4.3 and taking the adjoint in the equality (51), we obtain the strong stability of $T_{X^{+}}^{*}(t)$. Moreover, from the equality (53) we obtain that $T_{X^{-}}(-t)$, the semigroup generated by $-A_{X^{-}}$, is also strongly stable.

Lemma 4.9. Suppose that the state linear system $\Sigma(A, B, C)$ satisfies Assumptions 4.14.4. Then $X^{-}$is the unique nonpositive, self-adjoint solution of the CARE.

Proof. Consider the state linear system $\Sigma(-A, B, C)$. From Lemma 4.8 we have that $T_{X^{-}}(-t)$, the semigroup generated by $-A_{X^{-}}$, is strongly stable. Applying Theorem 3.6 we obtain that $-X^{-}$is the unique self-adjoint nonnegative solution for the CARE associated to the newly defined system. In other words, $X^{-}$is the unique self-adjoint nonpositive solution for the CARE associated with the initial system.

We end this section with our first result on the ordering of solutions of the CARE.

Theorem 4.10. Suppose that Assumptions 4.1-4.4 hold. Then any self-adjoint solution $X$ of the CARE satisfies

$$
X^{-} \leq X \leq X^{+}
$$


Proof. Under the Assumptions 4.1-4.4, from Remark 4.1, Remark 4.2 and Lemma 4.9 we have that $X^{+}$and $X^{-}$are the unique nonnegative and nonpositive self-adjoint solutions of the CARE, respectively.

Since $X^{+}$is a solution of the CARE, we can use Lemma 2.6 with the control $u^{+}(t)=$ $-B^{*} X^{+} z(t)$ to obtain

$$
0 \leq J_{\left[0, t_{1}\right]}\left(z_{0}, u^{+}\right)=\left\langle z_{0}, X^{+} z_{0}\right\rangle-\left\langle z\left(t_{1}\right), X^{+} z\left(t_{1}\right)\right\rangle
$$

For any other self-adjoint solution of the CARE, we have by the same lemma that

$$
J_{\left[0, t_{1}\right]}\left(z_{0}, u^{+}\right)=\left\langle z_{0}, X z_{0}\right\rangle-\left\langle z\left(t_{1}\right), X z\left(t_{1}\right)\right\rangle+\int_{0}^{t_{1}}\left\|B^{*}\left(X-X^{+}\right) z(t)\right\|^{2} d t
$$

Since $X^{+}$is a strongly stabilizing solution, see Theorem 3.5, we obtain that $z\left(t_{1}\right)$ converges to zero as $t_{1} \rightarrow \infty$. Thus,

$$
\left\langle z_{0}, X z_{0}\right\rangle+\int_{0}^{\infty}\left\|B^{*}\left(X-X^{+}\right) z(t)\right\|^{2} d t=\left\langle z_{0}, X^{+} z_{0}\right\rangle
$$

for all $z_{0} \in Z$, and so $X \leq X^{+}$.

To prove the other inequality we note that $X$ is a solution of the CARE for the system $\Sigma(A, B, C)$ if and only if $-X$ is a solution of the the CARE associated to the system $\Sigma(-A, B, C)$. From Lemma's 4.6 and 4.8 we have that $-X^{-}$is a (strongly) stabilizing solution of the CARE associated to the system $\Sigma(-A, B, C)$. Using the first part of the proof, we conclude that $-X \leq-X^{-}$. Thus $X^{-} \leq X$

In the rest of the paper we assume that Assumptions 4.1-4.4 hold. Consequently, we have the existence of $X^{+}$and $X^{-}$, the maximal and the minimal solution of the CARE, respectively. Define

$$
\Delta:=X^{+}-X^{-}=X^{+}+\left(Y^{+}\right)^{-1}
$$

Note that by Remark A.7 we have that $\Delta$, the gap between the maximal and the minimal solution of the CARE, is coercive.

\subsection{Invariant subspaces}

In this section we present some results on semigroup-invariant spaces. 
We introduce two subspaces of the Hilbert space $Z$.

$$
\begin{aligned}
& \mathcal{S}^{+}(A):=\left\{z_{0} \in Z \mid \lim _{t \rightarrow \infty} T(t) z_{0}=0\right\} \\
& \mathcal{S}^{-}(A):=\left\{z_{0} \in Z \mid \lim _{t \rightarrow-\infty} T(t) z_{0}=0\right\}
\end{aligned}
$$

The subspaces we have just defined are $T(t)$-invariant, as we can easily check. The subspace $\mathcal{S}^{+}(A)$ consists of all stable states and the subspace $\mathcal{S}^{-}(A)$ of all anti-stable states.

We also define

$$
\Delta^{+}(X):=X^{+}-X
$$

for any $X \in \mathcal{L}(Z)$. We give an equivalent condition under which $X \in \mathcal{L}(Z)$ is also a solution of the CARE.

Lemma 4.11. A self-adjoint $X \in \mathcal{L}(Z)$ is a solution of the $C A R E$ if and only if

$$
A_{X^{+}}^{*} \Delta^{+}(X)+\Delta^{+}(X) A_{X^{+}}=-\Delta^{+}(X) B B^{*} \Delta^{+}(X) \text { on } D(A) .
$$

Proof. Necessity: We know that $X^{+}$is a self-adjoint solution of the CARE and let $X \in$ $\mathcal{L}(Z)$ be another self-adjoint solution of the CARE. This means that

$$
\begin{aligned}
& A^{*} X^{+}+X^{+} A-X^{+} B B^{*} X^{+}+C^{*} C=0 \text { on } D(A), \text { and } \\
& A^{*} X+X A-X B B^{*} X+C^{*} C=0 \text { on } D(A) .
\end{aligned}
$$

Subtracting the second equation from the first equation, we obtain

$$
\begin{aligned}
0= & A^{*}\left(X^{+}-X\right)+\left(X^{+}-X\right) A-X^{+} B B^{*} X^{+}+X B B^{*} X \\
= & A_{X^{+}}^{*}\left(X^{+}-X\right)+\left(X^{+}-X\right) A_{X^{+}}-X^{+} B B^{*} X \\
& +X^{+} B B^{*} X^{+}-X B B^{*} X^{+}+X B B^{*} X \\
= & A_{X^{+}}^{*} \Delta^{+}(X)+\Delta^{+}(X) A_{X^{+}}+\Delta^{+}(X) B B^{*} \Delta^{+}(X) \text { on } D(A),
\end{aligned}
$$

where $\Delta^{+}(X)$ is defined in (15). This means that the equality (16) holds.

Sufficiency: We use that $X^{+}$is a self-adjoint solution of the CARE to subtract (17) from (16) to obtain that $X$ satisfies (18).

Now, for an arbitrary solution $X \in \mathcal{L}(Z)$ we consider the subspace spanned by the stable states and the subspace spanned by the anti-stable states. Recall also the notation $A_{X}:=A-B B^{*} X$. It appears, that for a state in the subspace $\mathcal{S}^{+}\left(A_{X}\right)$ the closed loop 
feedback governed by $u(t)=-B^{*} X z(t)$ results in the same solution as the closed-loop feedback governed by $u^{+}(t)=-B^{*} X^{+} z(t)$. For a state in the subspace $\mathcal{S}^{-}\left(A_{X}\right)$ a similar result holds.

Lemma 4.12. Let $X \in \mathcal{L}(Z)$ be a solution of the CARE. Then,

a. $\left(X^{+}-X\right) z_{0}=0$ for all $z_{0} \in \mathcal{S}^{+}\left(A_{X}\right)$ and $\left(X^{-}-X\right) z_{0}=0$ for all $z_{0} \in \mathcal{S}^{-}\left(A_{X}\right)$,

b. $T_{X}(t) z_{0}=T_{X^{+}}(t) z_{0}$, for all $z_{0} \in \operatorname{cl}\left(\mathcal{S}^{+}\left(A_{X}\right)\right)$ and all $t \in \mathbb{R}$ and

$T_{X}(t) z_{0}=T_{X^{-}}(-t) z_{0}$, for all $z_{0} \in \operatorname{cl}\left(\mathcal{S}^{-}\left(A_{X}\right)\right)$ and all $t \in \mathbb{R}$, where $T_{X^{-}}(-t)$ is the group generated by $-A_{X^{-}}$

c. $\mathcal{S}^{+}\left(A_{X}\right)$ and $\mathcal{S}^{-}\left(A_{X}\right)$ are closed subspaces of $Z$.

Proof. a. Consider the cost criterium $J_{\left[0, t_{1}\right]}\left(z_{0}, u\right)$ associated with the system $\Sigma(A, B, C)$ as in (4). For any initial data $z_{0} \in \mathcal{S}^{+}\left(A_{X}\right)$ and the feedback law $u(t):=-B^{*} X z(t)$, we have that $\lim _{t_{1} \rightarrow \infty} z\left(t_{1}\right)=0$. We use now Lemma 2.6 twice (for $X$ and for $X^{+}$) to obtain that

$$
\begin{gathered}
J_{\left[0, t_{1}\right]}\left(z_{0}, u\right)=\left\langle z_{0}, X z_{0}\right\rangle-\left\langle z\left(t_{1}\right), X z\left(t_{1}\right)\right\rangle \text { and } \\
J_{\left[0, t_{1}\right]}\left(z_{0}, u\right)=\left\langle z_{0}, X^{+} z_{0}\right\rangle-\left\langle z\left(t_{1}\right), X^{+} z\left(t_{1}\right)\right\rangle+\int_{0}^{t_{1}}\left\|B^{*}\left(X^{+}-X\right) z(t)\right\|^{2} d t .
\end{gathered}
$$

Taking the limit as $t_{1} \rightarrow \infty$ in these equalities, we obtain that

$$
\left\langle z_{0}, X z_{0}\right\rangle=\left\langle z_{0}, X^{+} z_{0}\right\rangle+\int_{0}^{\infty}\left\|B^{*}\left(X^{+}-X\right) z(t)\right\|^{2} d t
$$

So, $\left\langle z_{0}, X z_{0}\right\rangle \geq\left\langle z_{0}, X^{+} z_{0}\right\rangle$ for all $z_{0} \in \mathcal{S}^{+}\left(A_{X}\right)$. From Theorem 4.10 we know that $X^{+} \geq X$, thus $\left\langle z_{0},\left(X^{+}-X\right) z_{0}\right\rangle=0$ for all $z_{0} \in \mathcal{S}^{+}\left(A_{X}\right)$. Because $X^{+}-X \geq 0$, we conclude that

$$
\left(X^{+}-X\right) z_{0}=0 \text { for all } z_{0} \in \mathcal{S}^{+}\left(A_{X}\right)
$$

Using similar arguments for it can be proved that $\left(X^{-}-X\right) z_{0}=0$ for all $z_{0} \in \mathcal{S}^{-}\left(A_{X}\right)$. $b$. Since $X$ and $X^{+}$both satisfy the CARE, we find that $-A_{X^{+}}+A_{X}=B B^{*}\left(X^{+}-X\right)$. Thus the difference is in $\mathcal{L}(Z)$. From $a$. we have that $B B^{*}\left(X^{+}-X\right)$ is zero on the $T_{X}(t)$-invariant subspace $\mathcal{S}^{+}\left(A_{X}\right)$. Using Lemma B.1, we conclude that the groups $T_{X}(t)$ and $T_{X^{+}}(t)$ are equal on $\operatorname{cl}\left(\mathcal{S}^{+}\left(A_{X}\right)\right)$. The equality of the groups $T_{X}(t)$ and $T_{X^{-}}(-t)$ on $\operatorname{cl}\left(\mathcal{S}^{-}\left(A_{X}\right)\right)$ can be proved similarly. 
c. Let $z_{0}$ be an element of $\operatorname{cl}\left(\mathcal{S}^{+}\left(A_{X}\right)\right)$. According to $b$., for all $t \in \mathbb{R}$ we have that $T_{X}(t) z_{0}$ and $T_{X^{+}}(t) z_{0}$ are equal. Since $T_{X^{+}}(t)$ is strongly stable, we obtain that

$$
\lim _{t \rightarrow \infty} T_{X}(t) z_{0}=\lim _{t \rightarrow \infty} T_{X^{+}}(t) z_{0}=0
$$

Consequently $z_{0} \in \mathcal{S}^{+}\left(A_{X}\right)$ and hence $\mathcal{S}^{+}\left(A_{X}\right)$ is closed. Similarly, we can prove that $\mathcal{S}^{-}\left(A_{X}\right)$ is closed.

Interesting geometrical relations between a $T_{X^{+}}(t)$-invariant and a $T_{X^{-}}(-t)$-invariant subspace is stated in the following two results. Recall that $T_{X^{-}}(-t)$ is the semigroup generated by $-A_{X^{-}}$.

Lemma 4.13. Let $S_{1}$ be a closed linear subspace of a Hilbert space $Z$, and define $S_{2}:=$ $\Delta^{-1} S_{1}^{\perp}$. Then the following two statements are equivalent.

1. $S_{1}$ is $T_{X^{+}}(t)$-invariant,

2. $S_{2}$ is $T_{X^{-}}(-t)$-invariant.

Proof. From Lemma A.8 we have that

$$
-A_{X^{-}}=\Delta^{-1} A_{X^{+}}^{*} \Delta
$$

which implies that

$$
T_{X^{-}}(-t)=\Delta^{-1} T_{X^{+}}^{*}(t) \Delta .
$$

We have that $S_{1}$ is $T_{X^{+}}(t)$-invariant is equivalent to $S_{1}^{\perp}$ is $T_{X^{+}}^{*}(t)$-invariant (see [6], Exercise 2.30.a, page 97) which is equivalent to $S_{2}$ is $\Delta^{-1} T_{X^{+}}^{*}(t) \Delta$-invariant. Using equality (19), the last statement is the same with saying that $S_{2}$ is $T_{X^{-}}(-t)$-invariant.

Theorem 4.14. a. Let $S_{1}$ be a closed $T_{X^{+}}(t)$-invariant linear subspace of $Z$, and $S_{2}:=$ $\Delta^{-1} S_{1}^{\perp}$. Then there exists a projection $P \in \mathcal{L}(Z)$ such that for each $z \in Z$

$$
z=P z+(I-P) z, \text { with } P z \in S_{1},(I-P) z \in S_{2} .
$$

For this projection we have:

$$
\begin{aligned}
T_{X^{+}}(t) P & =P T_{X^{+}}(t) P, \text { for all } t \geq 0, \\
P T_{X^{-}}(-t) & =P T_{X^{-}}(-t) P, \text { for all } t \geq 0, \\
P & =\Delta^{-1} P^{*} \Delta P .
\end{aligned}
$$


b. Conversely, if for a projection $P \in \mathcal{L}(Z)$ the relations (21) and (23) hold (or (22) and (23)), then we have:

$$
\begin{aligned}
& S_{1}:=\operatorname{ran}(P) \text { is } T_{X^{+}}(t) \text {-invariant } \\
& S_{2}:=\operatorname{ran}(I-P) \text { is } T_{X^{-}}(-t) \text {-invariant } \\
& S_{2}=\Delta^{-1} S_{1}^{\perp} .
\end{aligned}
$$

Proof. a. From Lemma B.3 we have that $Z=S_{1} \oplus S_{2}$. So we can define a projection $P \in \mathcal{L}(Z)$ such that

$$
z=P z+(I-P) z
$$

with $P z \in S_{1}$ and $(I-P) z \in S_{2}$. We prove that $P$ satisfies the equalities (21)-(23). The subspace $S_{1}$ is $T_{X^{+}}(t)$-invariant and $S_{1}=\operatorname{ran}(P)$. Then $T_{X^{+}}(t) P z \in S_{1}$ implies that $P T_{X^{+}}(t) P z=T_{X^{+}}(t) P z$ for all $t \geq 0$, which is the equality (21). Similarly, we prove the equality (22). Since $S_{1}$ is $T_{X^{+}}(t)$-invariant and $S_{2}:=\Delta^{-1} S_{1}^{\perp}$, Lemma 4.13 implies that $S_{2}$ is $T_{X^{-}}(-t)$-invariant. Moreover, $S_{2}=\operatorname{ran}(I-P)$. Then $T_{X^{-}}(-t)(I-P) z \in S_{2}$ implies that $(I-P) T_{X^{-}}(-t)(I-P) z=T_{X^{-}}(-t)(I-P) z$ or, equivalently, $P T_{X^{-}}(-t)=P T_{X^{-}}(-t) P$, which is the equality $(22)$.

Further we prove the equality (23). We rewrite the equality $S_{2}=\Delta^{-1} S_{1}^{\perp}$ as

$$
0=\langle\Delta(I-P) x, P y\rangle=\left\langle x,(I-P)^{*} \Delta P y\right\rangle=\left\langle P^{*} \Delta(I-P) x, y\right\rangle
$$

for all $x, y \in Z$. This means that the equality (23) holds.

b. Assume that $P \in \mathcal{L}(Z)$ is a projection such that (21) and (23) hold. Define $S_{1}:=\operatorname{ran}(P)$ and $S_{2}:=\operatorname{ran}(I-P)$. We prove first that $S_{2}=\Delta^{-1} S_{1}^{\perp}$. Let us consider $s_{1} \in S_{1}$ and $s_{2} \in S_{2}$. Then, using (23), we obtain

$$
\begin{aligned}
\left\langle\Delta s_{2}, s_{1}\right\rangle & =\left\langle\Delta(I-P) s_{2}, P s_{1}\right\rangle=\left\langle s_{2},(I-P)^{*} \Delta P s_{1}\right\rangle \\
& =\left\langle s_{2}, \Delta\left(P-\Delta^{-1} P^{*} \Delta P\right) s_{1}\right\rangle=0
\end{aligned}
$$

and so $\Delta s_{2} \in S_{1}^{\perp}$, for all $s_{2} \in S_{2}$. Thus

$$
S_{2} \subset \Delta^{-1} S_{1}^{\perp}
$$

Now let us consider $x \in \Delta^{-1} S_{1}^{\perp}$. Then $x=\Delta^{-1} s_{1}^{\perp}$ for some $s_{1}^{\perp} \in S_{1}^{\perp}$. For arbitrary $z \in Z$ 
we have that

$$
\begin{aligned}
\langle P x, z\rangle & =\left\langle P \Delta^{-1} s_{1}^{\perp}, z\right\rangle=\left\langle s_{1}^{\perp}, \Delta^{-1} P^{*} z\right\rangle=\left\langle s_{1}^{\perp}, \Delta^{-1}\left(P^{*} \Delta\right) \Delta^{-1} z\right\rangle \\
& =\left\langle s_{1}^{\perp}, \Delta^{-1}\left(P^{*} \Delta P\right) \Delta^{-1} z\right\rangle=\left\langle s_{1}^{\perp}, P \Delta^{-1} z\right\rangle=0 .
\end{aligned}
$$

Consequently, we must have that $P x=0$, that is $(I-P) x=x$. This means that $x \in S_{2}$, and so

$$
\Delta^{-1} S_{1}^{\perp} \subset S_{2}
$$

Combining (27) and (28), we conclude that (26) holds.

From the assumption that (21) holds, it follows that for all $s_{1} \in S_{1}$ we have

$$
T_{X^{+}}(t) s_{1}=T_{X^{+}}(t) P s_{1}=P T_{X^{+}}(t) P s_{1},
$$

so (24) holds. From this and Lemma 4.13 we obtain (25).

In a completely similar way we can prove that if (22) and (23) hold, then (24), (25) and (26) also must hold.

\subsection{The main result}

Now we have all necessary ingredients to state and prove the main theorem of this paper, which is a generalization to infinite-dimensional systems of a well-known result of Willems $[21]$.

Theorem 4.15. Let $S_{1}$ be a closed $T_{X^{+}}(t)$-invariant subspace, and let $P \in \mathcal{L}(Z)$ be the projection on $S_{1}$ along the $T_{X^{-}}(-t)$-invariant subspace $S_{2}=\Delta^{-1} S_{1}^{\perp}$, then $X$ defined as

$$
X:=X^{+} P+X^{-}(I-P)
$$

is a solution of the CARE. Furthermore, $\mathcal{S}^{+}\left(A_{X}\right)=S_{1}, \mathcal{S}^{-}\left(A_{X}\right)=S_{2}$, and

$$
Z=\mathcal{S}^{+}\left(A_{X}\right) \oplus \mathcal{S}^{-}\left(A_{X}\right)
$$

Conversely, let $X$ be a self-adjoint solution of the CARE. Then (30) holds and $X$ can be decomposed as

$$
X=X^{+} P+X^{-}(I-P),
$$

where $P \in \mathcal{L}(Z)$ is the projection on the $T_{X^{+}}(t)$-invariant subspace $\mathcal{S}^{+}\left(A_{X}\right)$ along the 
$T_{X^{-}}(-t)$-invariant subspace $\mathcal{S}^{-}\left(A_{X}\right)$. Moreover,

$$
\mathcal{S}^{-}\left(A_{X}\right)=\Delta^{-1} \mathcal{S}^{+}\left(A_{X}\right)^{\perp}
$$

Proof. Step 1: Let $S_{1}$ be a closed $T_{X^{+}}(t)$-invariant subspace. In Lemma 4.13 we showed that $S_{2}=\Delta^{-1} S_{1}^{\perp}$ is a $T_{X^{-}}(-t)$-invariant subspace. Furthermore, Lemma 4.14 gives the existence of a projection $P$ satisfying (20)-(23). We now prove that $X$ defined by (29) and $P$ is a solution of the CARE.

Step 2: From Lemma 4.11, we know that $X$ is a solution of the CARE if and only if (16) holds. For $X$ defined by $(29)$ we can write $\Delta^{+}(X)$ as

$$
\Delta^{+}(X)=X^{+}-X=X^{+}-X^{+} P-X^{-}(I-P)=\Delta(I-P) .
$$

Substituting this expression for $\Delta^{+}(X)$ in (16), we obtain that $X$ is a solution of the CARE if and only if

$$
A_{X^{+}}^{*} \Delta(I-P)+\Delta(I-P) A_{X^{+}}+\Delta(I-P) B B^{*} \Delta(I-P)=0 \text { on } D(A) .
$$

Further we use the equality (53) to rewrite (33) as

$$
0=-A_{X^{-}}(I-P)+(I-P) A_{X^{+}}+(I-P) B B^{*} \Delta(I-P) \text { on } D(A) .
$$

We use the obvious equality

$$
B B^{*} \Delta=A_{X^{-}}-A_{X^{+}} \text {on } D(A)
$$

to rewrite (34) as

$$
0=-A_{X^{-}}(I-P)+(I-P) A_{X^{+}}+(I-P)\left(A_{X^{-}}-A_{X^{+}}\right)(I-P) \text { on } D(A) \text {. }
$$

So it suffices to prove the two equalities

$$
\begin{aligned}
& A_{X^{-}}(I-P)=(I-P) A_{X^{-}}(I-P) \text { on } D(A), \\
& (I-P) A_{X^{+}}=(I-P) A_{X^{+}}(I-P) \text { on } D(A) .
\end{aligned}
$$


Using relation $(22)$, i.e., $P T_{X^{-}}(-t)=P T_{X^{-}}(-t) P$ for all $t \geq 0$, we obtain that

$$
\begin{aligned}
(I-P) T_{X^{-}}(-t)(I-P) & =T_{X^{-}}(-t)-T_{X^{-}}(-t) P-P T_{X^{-}}(-t)+P T_{X^{-}}(-t) P \\
& =T_{X^{-}}(-t)-T_{X^{-}}(-t) P \\
& =T_{X^{-}}(-t)(I-P) .
\end{aligned}
$$

This means that

$$
\frac{d}{d t} T_{X^{-}}(-t)(I-P)=\frac{d}{d t}(I-P) T_{X^{-}}(-t)(I-P)
$$

so

$$
-A_{X^{-}} T_{X^{-}}(-t)(I-P)=(I-P)\left(-A_{X^{-}}\right) T_{X^{-}}(-t)(I-P) .
$$

For $t=0$ this becomes the equality (36). Starting with relation (21), we can prove (37) in a similar manner. Thus, the equality (16) holds, and using Lemma 4.11, we know that $X$ is a solution of the CARE.

Step 3: Next we prove that $\mathcal{S}^{+}\left(A_{X}\right)=S_{1}$. Assume first that $z \in \mathcal{S}^{+}\left(A_{X}\right)$. Since $X$ is a solution of the CARE, from Lemma 4.12.a we have that $X z=X^{+} z$. Using the decomposition $Z=S_{1} \oplus S_{2}$, we can write $z=z_{1}+z_{2}$, where $z_{1} \in S_{1}$ and $z_{2} \in S_{2}$. Then

$$
X z=X^{+} z=X^{+} z_{1}+X^{+} z_{2} .
$$

From the representation (29) we can write

$$
X z=X^{+} z_{1}+X^{-} z_{2}
$$

Using (38) we obtain

$$
\left(X^{+}-X^{-}\right) z_{2}=0
$$

and the coercivity of $\Delta=X^{+}-X^{-}$implies that $z_{2}=0$. This means that $z=z_{1} \in S_{1}$, and so we have proved that $\mathcal{S}^{+}\left(A_{X}\right) \subseteq S_{1}$. To prove the other inclusion we start with the following observation

$$
\left(A_{X}-A_{X^{+}}\right)=-B B^{*}\left(X-X^{+}\right)
$$

and so the difference of the two generators is bounded. Furthermore, for $s_{1} \in S_{1}$ we have that

$$
B B^{*}\left(X-X^{+}\right) s_{1}=B B^{*}\left(X-X^{+}\right) P s_{1}=0,
$$

where we have used (29). Finally, since $S_{1}$ a closed $T_{X^{+}}(t)$-invariant subspace of $Z$, we 
have by Lemma B.1 that $T_{X}(t) s_{1}=T_{X^{+}}(t) s_{1}$ for all $s_{1} \in S_{1}$. Since $T_{X^{+}}(t)$ is strongly stable, it follows that $s_{1} \in \mathcal{S}^{+}\left(A_{X}\right)$. Concluding, we have that $S_{1} \subseteq S^{+}\left(A_{X}\right)$, and thus $\mathcal{S}^{+}\left(A_{X}\right)=S_{1}$. Similarly, it can be proved that $\mathcal{S}^{-}\left(A_{X}\right)=S_{2}$, and so the equality (30) holds.

Step 4: Let $X$ be a self-adjoint solution of the CARE. From Lemma 4.12.c we have that $\mathcal{S}^{+}\left(A_{X}\right)$ is a closed subspace of $Z$. It is easy to see that $\mathcal{S}^{+}\left(A_{X}\right)$ is $T_{X}(t)$-invariant, and using Lemma 4.12.b we obtain that $\mathcal{S}^{+}\left(A_{X}\right)$ is $T_{X^{+}}(t)$-invariant. Take $S_{1}:=\mathcal{S}^{+}\left(A_{X}\right)$ in the first part of this theorem. Let $P \in \mathcal{L}(Z)$ be the projection on $S_{1}$ along the $T_{X^{-}}(-t)$ invariant subspace $S_{2}:=\Delta^{-1} S_{1}^{\perp}$. Thus, from the first part of this theorem, $\tilde{X}$ defined as

$$
\widetilde{X}:=X^{+} P+X^{-}(I-P)
$$

is a solution of the CARE and

$$
Z=S_{1} \oplus S_{2}=\mathcal{S}^{+}\left(A_{\tilde{X}}\right) \oplus \mathcal{S}^{-}\left(A_{\tilde{X}}\right)
$$

From Lemma 4.12.a we know that $X=X^{+}$on $S_{1}=\mathcal{S}^{+}\left(A_{X}\right)$ and by definition $\widetilde{X}=X^{+}$ on $S_{1}=\mathcal{S}^{+}\left(A_{\tilde{X}}\right)$. Thus $X=\tilde{X}=X^{+}$on $S_{1}=\mathcal{S}^{+}\left(A_{\tilde{X}}\right)=\mathcal{S}^{+}\left(A_{X}\right)$. Recall that, by the definition of $\tilde{X}$, we have that $\tilde{X}=X^{-}$on $S_{2}=\Delta^{-1} S_{1}^{\perp}=\mathcal{S}^{-}\left(A_{\tilde{X}}\right)$, and from Lemma 4.12.a, $X=X^{-}$on $\mathcal{S}^{-}\left(A_{X}\right)$.

Step 5: It remains to show that $X=\widetilde{X}\left(=X^{-}\right)$on $S_{2}$. For $A_{X^{-}}:=A-B B^{*} X^{-}$and $\Delta^{-}(X):=X-X^{-}$we can obtain the following analogue to Lemma $4.11 ; X$ is a solution to the CARE if and only if the following equation is satisfied

$$
A_{X^{-}}^{*} \Delta^{-}(X)+\Delta^{-}(X) A_{X^{-}}-\Delta^{-}(X) B B^{*} \Delta^{-}(X)=0 .
$$

$\widetilde{X}$ is also a solution to the CARE, and so

$$
A_{X^{-}}^{*} \Delta^{-}(\tilde{X})+\Delta^{-}(\tilde{X}) A_{X^{-}}-\Delta^{-}(\tilde{X}) B B^{*} \Delta^{-}(\tilde{X})=0 .
$$

Subtracting (40) from (39) leads to

$$
A_{X^{-}}^{*}(X-\tilde{X})+(X-\tilde{X}) A_{X^{-}}=\Delta^{-}(X) B B^{*} \Delta^{-}(X)-\Delta^{-}(\tilde{X}) B B^{*} \Delta^{-}(\tilde{X}) .
$$

It is easy to see that the connection between $\Delta^{-}(\tilde{X})$ and $\Delta^{-}(X)$ is

$$
\Delta^{-}(\tilde{X})=\Delta^{-}(X)-Q
$$


where $Q:=(X-\tilde{X})(I-P)=\left(X-X^{-}\right)(I-P)$. Using (42) in (41), we obtain the equality

$$
A_{X^{-}}^{*}(X-\tilde{X})+(X-\tilde{X}) A_{X^{-}}=-Q B B^{*} Q+Q B B^{*} \Delta^{-}(X)+\Delta^{-}(X) B B^{*} Q
$$

Recall that $T_{X^{-}}(-t)$ is the semigroup generated by $-A_{X^{-}}$, and so

$$
\begin{aligned}
& \frac{d}{d t}\left\langle T_{X^{-}}(-t) z,(X-\tilde{X}) T_{X^{-}}(-t) y\right\rangle \\
& \quad=\left\langle T_{X^{-}}(-t) z,-\left[A_{X^{-}}^{*}(X-\widetilde{X})+(X-\widetilde{X}) A_{X^{-}}\right] T_{X^{-}}(-t) y\right\rangle .
\end{aligned}
$$

Integrating the above equality from $-t$ to $0, t>0$, we find

$$
\begin{aligned}
\langle z,(X & -\tilde{X}) y\rangle-\left\langle T_{X^{-}}(-t) z,(X-\tilde{X}) T_{X^{-}}(-t) y\right\rangle \\
& =\int_{-t}^{0}\left\langle T_{X^{-}}(s) z,-\left[A_{X^{-}}^{*}(X-\widetilde{X})+(X-\widetilde{X}) A_{X^{-}}\right] T_{X^{-}}(s) y\right\rangle d s \\
& =\int_{-t}^{0}\left\langle T_{X^{-}}(s) z,\left[Q B B^{*} Q-Q B B^{*} \Delta^{-}(X)-\Delta^{-}(X) B B^{*} Q\right] T_{X^{-}}(s) y\right\rangle d s
\end{aligned}
$$

where we have used (43). Theorem 4.14.a says also that the above defined projection $P$ satisfies $P T_{X^{-}}(s)=P T_{X^{-}}(s) P$ for all negative $s$, which is equivalent to $T_{X^{-}}(s)(I-P)=$ $(I-P) T_{X^{-}}(s)(I-P)$ for all negative $s$. This allows us to write

$$
\begin{aligned}
Q T_{X^{-}}(s)(I-P) x & =(X-\tilde{X})(I-P) T_{X^{-}}(s)(I-P) x \\
& =\left(X-X^{-}\right) T_{X^{-}}(s)(I-P) x \\
& =\Delta^{-}(X) T_{X^{-}}(s)(I-P) x
\end{aligned}
$$

for any $x \in Z$. Taking in (44) $z=y=(I-P) x$ and using (45) we obtain that

$$
\begin{gathered}
\left\langle(I-P) x,\left(X-X^{-}\right)(I-P) x\right\rangle-\left\langle T_{X^{-}}(-t)(I-P) x,\left(X-X^{-}\right) T_{X^{-}}(-t)(I-P) x\right\rangle \\
=-\int_{-t}^{0}\left\|B^{*} \Delta^{-}(X) T_{X^{-}}(s)(I-P) x\right\|^{2} d s .
\end{gathered}
$$

Now $T_{X^{-}}(-t)(I-P) x \rightarrow 0$ as $t \rightarrow \infty$, and so

$$
\left\langle(I-P) x,\left(X-X^{-}\right)(I-P) x\right\rangle+\int_{-\infty}^{0}\left\|B^{*} \Delta^{-}(X) T_{X^{-}}(s)(I-P) x\right\|^{2} d s=0 .
$$

Since $X^{-}$is the minimal solution of the CARE, we conclude that $\left(X-X^{-}\right)(I-P) x=0$. In other words $X=X^{-}$on $S_{2}$. However, we also know that $\widetilde{X}=X^{-}$on $S_{2}$ and $X=\widetilde{X}=X^{+}$ 
on $S_{1}$. Consequently, $X=\tilde{X}$ and for any $X$ self-adjoint solution of the CARE we have that $Z=\mathcal{S}^{+}\left(A_{X}\right) \oplus \mathcal{S}^{-}\left(A_{X}\right)$. We also conclude that $S_{2}=\Delta^{-1} S_{1}^{\perp}=\mathcal{S}^{-}\left(A_{X}\right)$.

Corollary 4.16. For any $X$ self-adjoint solution of the CARE we have that

$$
Z=\mathcal{S}^{+}\left(A_{X}\right) \oplus \mathcal{S}^{-}\left(A_{X}\right)
$$

\section{Conclusions}

Infinite-dimensional systems is a well-established and popular area of research for both engineering and mathematics communities. One of the most important achievements in this field is that some optimal control problems may be solved analytically via the algebraic Riccati equation. Whereas the existence solutions of the AREs and the connection with stability properties of the closed-loop system seems to be reasonably well known, the behavior of numerical algorithms for solving an ARE corresponding to an infinite-dimensional system is not completely understood. The theoretical result derived in Theorem 4.15 might be of help in this direction (see also [21, Remark 20, page 630]). Moreover, there are certain area of applications, e.g. network synthesis realizations and inverse control problems where the knowledge of all solutions of the associated $\operatorname{ARE}(\mathrm{s})$ is of interest.

Our result of Theorem 4.15 extends the classification of all symmetric solutions of the CARE found, for a finite-dimensional systems, by Willems [21, Theorem 6, page 629] to the class of infinite dimensional systems which satisfies Assumptions 4.1-4.4. Similar results for different classes of infinite-dimensional systems can be found in the literature: in $[1,11]$ a separating spectra condition was assumed; in [10] the relation between the ARE and the eigenvectors of the Hamiltonian was studied for Riesz-spectral systems and extended in [17] for some classes of infinite-dimensional systems in connection with exponential stability of the closed-loop system. Under our assumptions the generator of the semigroup need not satisfy a separating spectra condition, or be Riesz-spectral, and the closed-loop might not be exponentially stable.

Future research needs to be done to relax the assumption that $A$ generates a group to $A$ generates a semigroup and to remove the invertibility assumption made on the minimal nonnegative self-adjoint solution of the Filter Riccati equation. Of course the case of unbounded $B$ and $C$ can be also investigated, and some results in this direction can be found in [15]. 


\section{A Appendix I: Invertible solutions of a Lyapunov equa- tion}

Using similar techniques as in the proof of Lemma 8.3.2.a in [6], we first prove a technical result which will be useful in the rest of this appendix.

Lemma A.1. Let $A_{1}$ and $A_{2}$ be two closed, linear operators on the Hilbert space $Z$. If $L \in \mathcal{L}(Z)$ is an invertible solution of the Lyapunov equation

$$
\left\langle A_{1} z_{1}, L z_{2}\right\rangle+\left\langle L^{*} z_{1}, A_{2} z_{2}\right\rangle=0
$$

for $z_{1} \in D\left(A_{1}\right), z_{2} \in D\left(A_{2}\right)$ and $\rho\left(-A_{1}^{*}\right) \cap \rho\left(A_{2}\right) \neq \emptyset$, then $L D\left(A_{2}\right)=D\left(A_{1}^{*}\right)$.

Proof. By the definition of $D\left(A_{1}^{*}\right)$ it is easy to see that the equation (46) implies that $L D\left(A_{2}\right) \subset D\left(A_{1}^{*}\right)$. Furthermore, the Lyapunov equation can be written as

$$
L A_{2}=-A_{1}^{*} L \text {, on } D\left(A_{2}\right) \text {. }
$$

Choose $s \in \rho\left(-A_{1}^{*}\right) \cap \rho\left(A_{2}\right)$. Then we may write the above equality as

$$
L\left(s I-A_{2}\right)=\left(s I+A_{1}^{*}\right) L, \text { on } D\left(A_{2}\right) .
$$

Since $\left(s I-A_{2}\right)$ and $\left(s I+A_{1}^{*}\right)$ are invertible, we obtain

$$
\left(s I+A_{1}^{*}\right)^{-1} L=L\left(s I-A_{2}\right)^{-1} \text {, on } Z \text {. }
$$

Consider the following list of equalities on $D\left(A_{1}^{*}\right)$ :

$$
\begin{aligned}
L^{-1} & =L^{-1}\left(s I+A_{1}^{*}\right)^{-1} L L^{-1}\left(s I+A_{1}^{*}\right) \\
& =L^{-1} L\left(s I-A_{2}\right)^{-1} L^{-1}\left(s I+A_{1}^{*}\right) \\
& =\left(s I-A_{2}\right)^{-1} L^{-1}\left(s I+A_{1}^{*}\right) .
\end{aligned}
$$

Thus we conclude that $L^{-1}$ maps the domain of $A_{1}^{*}$ into the domain of $A_{2}$. Since we already know that $L$ maps the domain of $A_{2}$ into the domain of $A_{1}^{*}$, we have proved the assertion.

Corollary A.2. Let $A$ be an infinitesimal generator of a $C_{0}$-semigroup on a Hilbert space $Z$, and let $Q$ be a bounded self-adjoint operator. Assume further that $L$ is a self-adjoint 
invertible solution of the controllability Lyapunov equation

$$
A L+L A^{*}=-Q \quad \text { on } D\left(A^{*}\right)
$$

and $\rho\left(-A-Q L^{-1}\right) \cap \rho\left(A^{*}\right) \neq \emptyset$. Then $L D\left(A^{*}\right)=D(A)$.

Proof. If we choose $A_{1}=A^{*}+L^{-1} Q$ and $A_{2}=A^{*}$, then equation (47) is the same as the equation (46). Hence we conclude from Lemma A.1 that

$$
L D\left(A^{*}\right)=L D\left(A_{2}\right)=D\left(A_{1}^{*}\right)=D(A)
$$

One might wonder whether the result of Lemma A.1 still holds when we remove the condition that $\rho\left(-A_{1}^{*}\right) \cap \rho\left(A_{2}\right) \neq \emptyset$. The following example shows that this is not true.

Example A.3. Let $A$ be the differentiation operator on $Z=L^{2}(0, \infty)$, i.e., $A h=d h / d x$, on its domain $D(A)=\left\{h \in L^{2}(0, \infty) \mid h\right.$ is absolutely continuous, and $\left.\frac{d h}{d x} \in L^{2}(0, \infty)\right\}$. It is well known and easy to see that the dual operator is given by $A^{*} f=-d f / d x$ on its domain $D\left(A^{*}\right)=\left\{f \in L^{2}(0, \infty) \mid f\right.$ is absolutely continuous, $f(0)=0$ and $\left.\frac{d f}{d x} \in L^{2}(0, \infty)\right\}$.

Choose $A_{1}=A_{2}=A^{*}$ and $L=I$. Then equation (46) holds. However,

$$
L D\left(A_{2}\right)=D\left(A^{*}\right) \subsetneq D(A)=D\left(A_{1}^{*}\right) .
$$

Thus we see that the conclusion of the Lemma A.1 does not hold. Looking at the spectrum of $A$, we see that this equals to the whole closed right-half plane. Thus $\rho\left(-A_{1}^{*}\right) \cap \rho\left(A_{2}\right)=$ $\rho(-A) \cap \rho(A)=\emptyset$.

Using Lemma A.1 we can derive several results, which will be used to prove Lemma 4.6 .

Lemma A.4. Suppose that $X^{+}$and $Y^{+}$are nonnegative solutions of the CARE and FARE associated to the state linear system $\Sigma(A, B, C)$. Then $I+Y^{+} X^{+}$is invertible on $D(A)$, i.e.,

$$
\left(I+Y^{+} X^{+}\right)^{-1} D(A)=D(A)
$$

Furthermore, $I+X^{+} Y^{+}$is invertible on $D\left(A^{*}\right)$, i.e.,

$$
\left(I+X^{+} Y^{+}\right)^{-1} D\left(A^{*}\right)=D\left(A^{*}\right)
$$


Proof. Since $X^{+}$and $Y^{+}$are nonnegative, we know that $\left(I+Y^{+} X^{+}\right)^{-1}$ is boundedly invertible. Since $X^{+} D(A) \subset D\left(A^{*}\right)$ and $Y^{+} D\left(A^{*}\right) \subset D(A)$, it is easy to see that $(I+$ $\left.Y^{+} X^{+}\right) D(A) \subset D(A)$. In order to apply Lemma A.1 we introduce the following perturbation of the infinitesimal generator $A$

$$
A_{2}:=A+M Y^{+} C^{*} C M^{-1}-B B^{*} X^{+}
$$

where $M:=\left(I+Y^{+} X^{+}\right)^{-1}$, and so $D\left(A_{2}\right)=D(A)$. On $D(A)$ we have the sequence of equalities

$$
\begin{aligned}
M^{-1} A_{2} & =M^{-1}\left(A+M Y^{+} C^{*} C M^{-1}-B B^{*} X^{+}\right) \\
& =M^{-1} A+Y^{+} C^{*} C M^{-1}-M^{-1} B B^{*} X^{+} \\
& =\left(I+Y^{+} X^{+}\right) A+Y^{+} C^{*} C\left(I+Y^{+} X^{+}\right)-\left(I+Y^{+} X^{+}\right) B B^{*} X^{+} \\
& =\left(I+Y^{+} X^{+}\right) A+Y^{+} C^{*} C+Y^{+} C^{*} C Y^{+} X^{+}-B B^{*} X^{+}-Y^{+} X^{+} B B^{*} X^{+} \\
& =\left(I+Y^{+} X^{+}\right) A+Y^{+}\left(C^{*} C-X^{+} B B^{*} X^{+}\right)+\left(Y^{+} C^{*} C Y^{+}-B B^{*}\right) X^{+} \\
& =\left(I+Y^{+} X^{+}\right) A+Y^{+}\left(-A^{*} X^{+}-X^{+} A\right)+\left(A Y^{+}+Y^{+} A^{*}\right) X^{+} \\
& =A\left(I+Y^{+} X^{+}\right) \\
& =A M^{-1}
\end{aligned}
$$

where we have used CARE, FARE, $X^{+}: D(A) \rightarrow D\left(A^{*}\right)$ and $Y^{+}: D\left(A^{*}\right) \rightarrow D(A)$. Thus

$$
M^{-1} A_{2} z=A M^{-1} z, \text { for } z \in D(A) .
$$

This is equivalent to

$$
\left\langle-A^{*} z_{1}, M^{-1} z_{2}\right\rangle+\left\langle M^{-*} z_{1}, A_{2} z_{2}\right\rangle=0
$$

for $z_{1} \in D\left(A^{*}\right), z_{2} \in D\left(A_{2}\right)$. If we define $A_{1}$ as $-A^{*}$, then the above equality is the same as (46). Since $-A_{1}^{*}=A$, and since $A_{2}$ is a bounded perturbation of $A$ their resolvent sets have a non-empty intersection. So from Lemma A.1 we conclude that $M^{-1} D(A)=$ $M^{-1} D\left(A_{2}\right)=D\left(A_{1}^{*}\right)=D(A)$. Thus $\left(I+Y^{+} X^{+}\right)^{-1} D(A)=D(A)$.

As a direct corollary of the above result we can now relate the closed loop generators of the CARE and FARE.

Lemma A.5. Suppose that $X^{+}$and $Y^{+}$are nonnegative solutions of the CARE and FARE 
associated with the state linear system $\Sigma(A, B, C)$. The following equality holds:

$$
A_{Y^{+}}=\left(I+Y^{+} X^{+}\right) A_{X^{+}}\left(I+Y^{+} X^{+}\right)^{-1} \text { on } D(A) \text {. }
$$

Proof. Note that we use the notations

$$
A_{Y^{+}}=A-Y^{+} C^{*} C \text { and } A_{X^{+}}=A-B B^{*} X^{+} .
$$

Using equation (50), we have that on $D(A)$

$$
\left(I+Y^{+} X^{+}\right)\left(A+M Y^{+} C^{*} C M^{-1}-B B^{*} X^{+}\right)=A\left(I+Y^{+} X^{+}\right)
$$

where $M=\left(I+Y^{+} X^{+}\right)^{-1}$. This is is equivalent to

$$
\left(A-Y^{+} C^{*} C\right)\left(I+Y^{+} X^{+}\right)=\left(I+Y^{+} X^{+}\right)\left(A-B B^{*} X^{+}\right) \text {on } D(A) .
$$

Using Lemma A.4 and multiplying by $\left(I+Y^{+} X^{+}\right)^{-1}$ to the right, we obtain the equality (51).

Remark A.6. Lemma A.5 and hold under Assumptions 4.1-4.2, see Remark 4.1 and Remark 4.2.

The next lemma concerns the "gap" $\Delta$. Suppose that the state linear system $\Sigma(A, B, C)$ satisfies the Assumptions 4.1-4.3. Let $\Delta$ be defined as

$$
\Delta:=X^{+}+\left(Y^{+}\right)^{-1}
$$

Remark A.7. The operator $\Delta$ defined in (52) is coercive. Indeed, since $Y^{+}$and $X^{+}$are nonnegative, $\left(\left(Y^{+}\right)^{\frac{1}{2}} X^{+}\left(Y^{+}\right)^{\frac{1}{2}}+I\right)$ is invertible. From (52) we have that

$$
\Delta=X^{+}+\left(Y^{+}\right)^{-1}=\left(Y^{+}\right)^{-\frac{1}{2}}\left(\left(Y^{+}\right)^{\frac{1}{2}} X^{+}\left(Y^{+}\right)^{\frac{1}{2}}+I\right)\left(Y^{+}\right)^{-\frac{1}{2}} .
$$

Consequently, $\Delta$ is invertible. Furthermore, it is clear that it is positive, hence we have that it is coercive.

Lemma A.8. Suppose that the state linear system $\Sigma(A, B, C)$ satisfies Assumptions 4.14.3, and $Y^{+} D\left(A^{*}\right)=D(A)$. Then the following equality holds:

$$
A_{X^{-}}=A-B B^{*}\left(-Y^{+}\right)^{-1}=-\Delta^{-1}\left(A_{X^{+}}\right)^{*} \Delta \text { on } D(A) \text {. }
$$


Proof. From (52), Lemma A.4, and the assumption $D\left(A^{*}\right)=\left(Y^{+}\right)^{-1} D(A)$ we have that

$$
\begin{aligned}
Y^{+} \Delta D(A) & =\left(I+Y^{+} X^{+}\right) D(A)=D(A), \\
\Delta^{-1} D\left(A^{*}\right) & =\left(I+Y^{+} X^{+}\right)^{-1} Y^{+} D\left(A^{*}\right)=D(A) .
\end{aligned}
$$

Using these domain properties in relation (51) gives

$$
A_{Y^{+}} Y^{+}=A Y^{+}-Y^{+} C^{*} C Y^{+}=Y^{+} \Delta\left(A-B B^{*} X^{+}\right) \Delta^{-1} \text { on } D\left(A^{*}\right) .
$$

Using that $Y^{+}$is a solution for the FARE, we obtain that

$$
Y^{+} A^{*}+B B^{*}=-Y^{+} \Delta\left(A-B B^{*} X^{+}\right) \Delta^{-1} \text { on } D\left(A^{*}\right) .
$$

Now, consider arbitrary $x \in D(A)$ and $y \in D\left(A^{*}\right)$. Since $Y^{+} D\left(A^{*}\right)=D(A)$, there exists $y_{0} \in D(A)$ such that $y=\left(Y^{+}\right)^{-1} y_{0}$. We have the following sequence of equivalences

$$
\begin{aligned}
& \left\langle\left(Y^{+} A^{*}+B B^{*}\right) x, y\right\rangle=-\left\langle Y^{+} \Delta A_{X^{+}} \Delta^{-1} x, y\right\rangle \\
\Longleftrightarrow & \left\langle x,\left(A Y^{+}+B B^{*}\right)\left(Y^{+}\right)^{-1} y_{0}\right\rangle=-\left\langle x, \Delta^{-1} A_{X^{+}}^{*} \Delta Y^{+}\left(Y^{+}\right)^{-1} y_{0}\right\rangle \\
\Longleftrightarrow & \left\langle x, A_{X^{-}} y_{0}\right\rangle=-\left\langle x, \Delta^{-1} A_{X^{+}}^{*} \Delta y_{0}\right\rangle
\end{aligned}
$$

for arbitrary $x \in D(A)$ and $y_{0} \in D(A)$. This means that the equality (53) holds.

\section{B Appendix II}

In this appendix we state several auxiliary results used throughout the paper.

The following lemma gives a sufficient condition for two generators that are equal on a subspace to generate equal groups on this subspace.

Lemma B.1. Let $A_{1}$ and $A_{2}$ be the infinitesimal generators of the $C_{0}$-groups $T_{1}(t)$ and $T_{2}(t)$, respectively. Assume that $D\left(A_{1}\right)=D\left(A_{2}\right), S:=A_{1}-A_{2} \in \mathcal{L}(Z)$ and that there exists a $T_{1}(t)$-invariant subspace $V$. If $S$ is zero on $V$, then the groups are equal on $\mathrm{cl}(V)$.

Proof. Since $S \in \mathcal{L}(Z)$ it follows that $S z=0$ for all $z \in \operatorname{cl}(V)$. Next, we prove that $\operatorname{cl}(V)$ is $T_{1}(t)$-invariant. For each $t \geq 0$, we have that $T_{1}(t) \in \mathcal{L}(Z)$. Let $z \in \operatorname{cl}(V)$. Then there exists a sequence $z_{n} \in V$ with $\lim _{n \rightarrow \infty} z_{n}=z$. Now, for each $t \geq 0$, we have that

$$
T_{1}(t) z=T_{1}(t) \lim _{n \rightarrow \infty} z_{n}=\lim _{n \rightarrow \infty} T_{1}(t) z_{n} \in \operatorname{cl}(V) .
$$


Consequently, $\operatorname{cl}(V)$ is $T_{1}(t)$-invariant.

Since $A_{2}=A_{1}-S$, we have by Theorem 3.2.1 of [6] that for positive $t$

$$
T_{2}(t) z=T_{1}(t) z-\int_{0}^{t} T_{2}(t-s) S T_{1}(s) z d s .
$$

For $z \in \mathrm{cl}(V)$, this immediately implies that $T_{2}(t) z=T_{1}(t) z$. Now we can repeat the same argument for the semigroups $\left.\left(T_{1}(-t)\right)_{t \geq 0}\right)$ and $\left.\left(T_{1}(-t)\right)_{t \geq 0}\right)$, and so we have that the groups $T_{1}(t)$ and $T_{2}(t)$ are equal on $\operatorname{cl}(V)$.

We consider the system (2) with the cost criterium on a finite time-interval

$$
J_{[a, b]}\left(z_{0}, u\right)=\int_{a}^{b}\left(\|y(s)\|^{2}+\|u(s)\|^{2}\right) d s
$$

for $0 \leq a \leq b$. We can rewrite the cost functional in a useful way. One can see that the following result is an extension of Lemma 2.6, and we will use it only in the proof of Theorem 4.12.d.

Lemma B.2. Let $X \in \mathcal{L}(Z)$ be a self-adjoint solution of the CARE. Consider the cost criterium on a finite time-interval (54), with $z$ and $u$ related via (2). The cost criterium can be written as

$$
J_{[a, b]}\left(z_{0} ; u\right)=\int_{a}^{b}\left\|u(s)+B^{*} X z(s)\right\|^{2} d s+\langle z(a), X z(a)\rangle-\langle z(b), X z(b)\rangle .
$$

Proof. For $u \in C^{1}(a, b ; U)$ and $z(a) \in D(A)$, we have

$$
\begin{aligned}
\langle z(a), X z(a)\rangle-\langle z(b), X z(b)\rangle= & -\int_{a}^{b} \frac{d}{d t}\langle z(t), X z(t)\rangle d t \\
= & -\int_{a}^{b}\left(\left\langle z(t),\left(A^{*} X+X A\right) z(t)\right\rangle\right. \\
& +\langle B u(t), X z(t)\rangle+\langle z(t), X B u(t)\rangle) d t .
\end{aligned}
$$


Using the CARE and the assumption that $X$ is self-adjoint we rewrite the integrand,

$$
\begin{aligned}
\left\langle z,\left(A^{*} X\right.\right. & +X A) z\rangle+\langle B u, X z\rangle+\langle z, X B u\rangle \\
& =-\left\langle z, C^{*} C z\right\rangle+\left\langle B^{*} X z, B^{*} X z\right\rangle+\langle B u, X z\rangle+\langle z, X B u\rangle \\
& =\left\|u+B^{*} X z\right\|_{R}^{2}-\langle y, y\rangle-\langle u, u\rangle .
\end{aligned}
$$

We see that $J_{[a, b]}\left(z_{0} ; u\right)$ is indeed as claimed for smooth inputs and initial conditions in the domain of $A$. Since these sets are dense in $L^{2}(a, b ; U)$ and $Z$, respectively, and since $(55)$ is well-defined on these sets, we conclude that the cost criterium can be written as (55).

The following result is needed in the proof of Theorem 4.14 .

Lemma B.3. Let $S_{1}$ be a closed linear subspace of a Hilbert space $Z$, and define $S_{2}:=$ $\Delta^{-1} S_{1}^{\perp}$, where $\Delta$ is a coercive, self-adjoint, bounded linear operator on $Z$. Then

$$
Z=S_{1} \oplus S_{2}
$$

Proof. Since $S_{1}$ is a linear subspace of $Z, S_{1}^{\perp}$ is a linear subspace as well and since $\Delta^{-1} \in$ $\mathcal{L}(Z), S_{2}$ is also a linear subspace of $Z$. Let the sequence $\left\{z_{n}\right\}_{n \in \mathbb{N}}$ be such that $z_{n} \in \Delta^{-1} S_{1}^{\perp}$ and $\lim _{n \rightarrow \infty} z_{n}=z \in Z$. Then $\left\langle\Delta z_{n}, s_{1}\right\rangle=0$, for all $s_{1} \in S_{1}$. The operator $\Delta$ is continuous, SO

$$
\left\langle\Delta z, s_{1}\right\rangle=\left\langle\Delta \lim _{n \rightarrow \infty} z_{n}, s_{1}\right\rangle=\lim _{n \rightarrow \infty}\left\langle\Delta z_{n}, s_{1}\right\rangle=0
$$

holds. Consequently, $z \in \Delta^{-1} S_{1}^{\perp}$. This means that $S_{2}$ is closed.

Clearly $S_{1}+S_{2}$ is a linear subspace of $Z$. Now we prove that $S_{1}+S_{2}$ is closed. We use the norm defined by $\|z\|_{\Delta}=\sqrt{\langle z, \Delta z\rangle}$. This norm is equivalent to the usual norm $\|z\|=\sqrt{\langle z, z\rangle}$, so we can use this norm in the definitions of a closed operator and a Cauchy sequence. Consider a Cauchy sequence $\left\{z_{n}\right\}_{n \in \mathbb{N}}$, such that $z_{n} \in S_{1}+S_{2}$ and $\lim _{n \rightarrow \infty} z_{n}=z \in Z$. Then $z_{n}=z_{n 1}+z_{n 2}$, with $z_{n 1} \in S_{1}$ and $z_{n 2} \in S_{2}$, for each $n$. For each $\varepsilon>0$ there must exist a $N_{0} \in \mathbb{N}$ such that for all $m, n>N_{0}$

$$
\varepsilon \geq\left\langle\left(z_{n}-z_{m}\right), \Delta\left(z_{n}-z_{m}\right)\right\rangle
$$

holds. Now we have:

$$
\begin{aligned}
\varepsilon & \geq\left\langle\left(z_{n}-z_{m}\right), \Delta\left(z_{n}-z_{m}\right)\right\rangle \\
& =\left\langle\left(z_{n 1}-z_{m 1}\right), \Delta\left(z_{n 1}-z_{m 1}\right)\right\rangle+\left\langle\left(z_{n 2}-z_{m 2}\right), \Delta\left(z_{n 2}-z_{m 2}\right)\right\rangle
\end{aligned}
$$


in which the cross-terms have disappeared, because $\Delta$ is self-adjoint and $S_{1}$ and $\Delta S_{2}$ are orthogonal. Both remaining terms are nonnegative, so we have

$$
\varepsilon \geq\left\langle\left(z_{n 1}-z_{m 1}\right), \Delta\left(z_{n 1}-z_{m 1}\right)\right\rangle
$$

Now $\left\{z_{n 1}\right\}_{n 1 \in \mathbb{N}}$ must be a Cauchy sequence in $S_{1}$. Because $S_{1}$ is closed, we have that $\lim _{n \rightarrow \infty} z_{n 1}=z_{1} \in S_{1}$. In the same way we derive $\lim _{n \rightarrow \infty} z_{n 2}=z_{2} \in S_{2}$. Now

$$
\lim _{n \rightarrow \infty} z_{n}=\lim _{n \rightarrow \infty}\left(z_{n 1}+z_{n 2}\right)=z_{1}+z_{2} \in S_{1}+S_{2},
$$

and so $S_{1}+S_{2}$ is closed.

Now we prove that $S_{1} \cap S_{2}=\{0\}$. Assume that $z \in S_{1} \cap S_{2}$. Then $\Delta z \in S_{1}^{\perp}$, so $\langle\Delta z, z\rangle=0$. Because $\Delta$ is coercive, self-adjoint we must have $z=0$.

Next we show that $S_{1}+S_{2}$ is dense in $Z$. By definition, $S_{1}+S_{2}$ is dense in $Z$ if the closure of $S_{1}+S_{2}$ is equal to $Z$. Assume that $z \in Z$ is such that $z \perp\left(S_{1}+S_{2}\right)$. Then $\left\langle z, s_{1}+\Delta^{-1} s_{1}^{\perp}\right\rangle=0$ for every $s_{1} \in S_{1}, s_{1}^{\perp} \in S_{1}^{\perp}$. In particular, for $s_{1}^{\perp}=0$, we have $\left\langle z, s_{1}\right\rangle=0$ for all $s_{1} \in S_{1}$, so $z \in S_{1}^{\perp}$. Thus it also holds for $s_{1}=0$, so we can choose $s_{1}^{\perp}=z$ and retrieve $\left\langle z, \Delta^{-1} z\right\rangle=0$. This means that $z=0$ and thus $S_{1}+S_{2}$ is dense in $Z$.

We have proved that $S_{1}+S_{2}$ is dense in $Z$ and that $S_{1}+S_{2}$ is a closed linear subspace of $Z$. From this we conclude that $S_{1}+S_{2}=Z$. Together with $S_{1} \cap S_{2}=\{0\}$ this implies that $S_{1} \oplus S_{2}=Z$.

\section{References}

[1] V. Adamjan, H. Langer and C. Tretter. Existence and Uniqueness of Contractive Solutions of some Riccati Equations. J. Funct. Anal. 179:2, 448-473, 2001.

[2] S. Bittanti, A.J. Laub, and J.C. Willems. The Riccati Equation. Springer Verlag, Berlin, 1991.

[3] F.M. Callier, L. Dumortier, and J. Winkin. On the nonnegative self-adjoint solutions of the operator riccati equation for infinite dimensional systems. Integral Equations and Operator Theory, 22(2):162-195, 1995.

[4] T. Chen and B. Francis. Spectral and inner-outer factorization of rational matrices. SIAM Journal of Matrix Analysis Applications, 10(1):1-17, 1989. 
[5] R.F. Curtain and L. Rodman. Comparison theorems for infinite-dimensional Riccati equations. Systems and Control Letters, 15(2):153-159, 1990.

[6] R.F. Curtain and H.J. Zwart. An Introduction to Infinite-Dimensional Linear Systems Theory. Springer-Verlag, New York, 1995.

[7] J.C. Doyle, K. Glover, P.P. Khorgonekar, and B.A. Francis. State-space solutions to standard $\mathrm{H}_{2}$ and $H_{\infty}$ control problems. IEEE Transactions on Automatic control, 34:831-847, 1989.

[8] P. Grabowski. On the spectral-Lyapunov approach to parametric optimization of distributed parameter systems. IMA Journal of Mathematical Control and Information, 7:317-338, 1991.

[9] S. Hansen and G. Weiss. New results on the operator Carleson measure criterion. IMA Journal of Mathematical Control and Information, 14:3-32, 1997.

[10] C.R. Kuiper and H.J. Zwart. Solutions of the ARE in terms of the Hamiltonian in terms of the Riesz spectral systems. In R.F. Curtain, A. Bensoussan, and J.L. Lions, editors, Analysis and Optimization of Systems: State and Frequancy Domain Approaches for Infinite Dimensional Systems, volume 185 of Lecture Notes in Control and Information Sciences, pages 314-325. Springer-Verlag, 1993.

[11] H. Langer, A.C.M. Ran and B.A. van de Rotten. Invariant subspaces of infinite dimensional Hamiltonians and solutions of the corresponding Riccati equations. In Operator Theory: Advances and Applications, Volume 130, Birkhäuser Verlag, 2001.

[12] J. Malinen. Discrete Time $H_{\infty}$-Algebraic Riccati Equations. Technical Report A428, Helsinki University of Technology, Espoo, Finland, 2000. Doctoral dissertation.

[13] K. Mikkola. Infinite-Dimensional Linear Systems, Optimal Control and Algebraic Riccati Equations. PhD thesis, Helsinki University of Technology, Institut of Mathematics, Finland, 2002.

[14] J.C. Oostveen. Strongly Stablizable Distributed Parameter Systems. Frontiers in Applied Mathematics, 20, SIAM, Philadelphia, 2000.

[15] M. Opmeer and O.V. Iftime. A Representation of All Bounded Selfadjoint Solutions of the Algebraic Riccati Equation for Systems with an Unbounded Observation Operator. The 43rd IEEE Conference on Decision and Control, Atlantis, Paradise Island, Bahamas, Dec. 14-17, 2004. 
[16] A. Pazy. Semigroups of Linear Operators and Applications to Partial Differential Equations. Springer Verlag, Berlin, 1983.

[17] J.J. Scholte. A classification of all solutions of the algebraic Riccati equation for infinite-dimensional systems. Technical report, University of Twente, 1999.

[18] O.J. Staffans. Quadratic optimal control of stable well-posed linear systems. Transactions of the American Mathematical Society, 349:3679-3716, 1997.

[19] O.J. Staffans. Coprime factorizations and well-posed linear systems. SIAM Journal on Control and Optimization, 36:1268-1292, 1998.

[20] P.A. van Dooren. Generalized approach for solving Riccati equations. SIAM J. Sci. St. Comp., 2(2):121-135, 1981.

[21] J.C. Willems. Least-squares stationary optimal control and the algebraic Riccati equation. IEEE Transactions on Automatic Control, 16:621-634, 1971.

[22] H. Zwart. On the invertibility and bounded extension of $C_{0}$-semigroups. Semigroup Forum, 63:153-160, 2001. 